\title{
Economía creativa, cultura y educación en tiempos de pandemia
}

Creative Economy, Culture and Education in Pandemic Times

\author{
Edna Dos Santos-Duisenberg \\ Consejera internacional en economía creativa \\ y políticas de desarrollo
}

En 2020 la pandemia de COVID-19 ha desestabilizado el mundo de una manera multidimensional. En cada esquina, hemos experimentado un cambio en el comportamiento humano y las actitudes diarias. De repente, el mundo ha pasado de la globalización al aislamiento. Desde abrazos y besos hasta distanciamiento social. Desde el contacto físico hasta los chats virtuales. Del gran consumismo hacia un mundo con mayor conciencia ambiental. Del egocentrismo hacia un enfoque centrado en el ser humano. En este contexto controvertido - la cultura, la creatividad y la conectividad - se han convertido en la columna vertebral de la sociedad, manteniendo unidas a las personas que estaban físicamente separadas ${ }^{1}$.

Las consecuencias económicas, sociales y culturales de esta pandemia son de largo alcance. La crisis de COVID-19 no solo

\footnotetext{
1 E. Dos Santos-Duisenberg, julio de 2020, «Brazilian culture and creativity during COVID-19: solidarity and lives»: https://www.pec.ac.uk/blog/brazilian-culture-and-creativity-during-covid19-solidarity-and-lives. 
nos ha robado más de 4,2 millones $^{2}$ de vidas en todo el mundo, sino también ha exacerbado la desigualdad, derribando la economía global y el libre comercio, destruyendo los sistemas nacionales de salud, el turismo y la vida urbana, y así agravando el desempleo y la inestabilidad social. Sin embargo, probablemente el legado positivo más profundo de esta situación caótica es el creciente sentido de solidaridad y ciudadanía que alienta a las personas a mejorar, participar y actuar.

\section{La emergencia socioeconómica}

En América Latina la pandemia ha hecho la desigualdad más visible. La región fue duramente golpeada por la COVID-19 registrando una caída del 7,4\% del PIB en 2020, con la pérdida de 26 millones de empleos la pobreza ha aumentado un 5\% en Latinoamérica. El paquete fiscal medio para hacer frente a la crisis fue del 8,5\% del PIB, el déficit fiscal sobrepasó el 5\% del PIB y la deuda pública alcanzo el $72 \%$ en $2020^{3}$. Fue el año más difícil de la historia moderna del continente.

Las industrias creativas y digitales, en particular el sector audiovisual, las redes sociales, las noticias en línea y los servicios de prensa y comunicaciones, han sido poderosos al mostrar la cruel realidad de la pobreza en el continente en el momento actual. Para los más vulnerables, el aislamiento social fue considerado un lujo. Es difícil estar en casa para evitar el contagio cuando no hay dinero para poder comer. Es difícil estar confinado en un aislamiento social cuando una gran familia vive en una habitación pequeña en las comunidades de barrios pobres. Es difícil lavarse las manos varias veces al día y tener prácticas de higiene cuando no hay agua y tampoco las condiciones sanitarias ade-

2 Organización Mundial de la Salud, situación el 2 de agosto de 2021: https://covid19.who.int. 3 Informe del Banco Interamericano de Desarrollo, marzo de 2021: https://flagships.iadb.org/ es/MacroReport2021/Oportunidades-para-un-mayor-crecimiento-sostenible-tras-la-pandemia. 
cuadas en el hogar. En estas circunstancias, algunos de los gobiernos latinos han asignado del presupuesto público nacional para implementar paquetes de emergencia COVID-19.

En Brasil el auxilio de emergencia ha incluido medidas fiscales y monetarias para ayudar a las pequeñas y medianas empresas (pymes), microempresas y trabajadores independientes. El esquema de cupones COVID-19 ha tenido una amplia cobertura en 2020; 65 millones de beneficiarios han seguido las instrucciones de elegibilidad digital y recibieron asistencia financiera, inicialmente durante cinco meses como compensación por sus pérdidas de ingresos ${ }^{4}$. Pero como la pandemia ha continuado alargándose, en marzo de 2021 el gobierno brasileño ha decidido extender la ayuda de emergencia hasta junio de este año. En suma, los gastos gubernamentales para mitigar la propagación continua de la pandemia ha alcanzado $\mathrm{R} \$ 1$ trillón en $2020^{5}$.

Cabe señalar que la digitalización y los servicios creativos (en forma de una aplicación móvil oficial) han hecho posible que el gobierno de un país de tamaño continental como Brasil establezca en un tiempo relativamente corto un programa masivo de distribución de renta. El esquema no solo ha capturado 13 millones de personas desempleadas, más 3,7 millones de trabajadores informales, sino también a los trabajadores independientes que no tienen ingresos y a aquellos que antes eran completamente invisibles (incluso del esquema de reducción de la pobreza familiar que cubre a 15 millones de familias). Con suerte, en el futuro esta gran colecta de datos pueda ser utilizada para diseñar políticas públicas apropiadas y programas educativos y culturales de capacitación más efectivos para abordar la falta de inclusión económica y social. En este contexto, las acti-

4 Ley 13.982 de 2020 del Gobierno Federal: https://www.gov.br/planalto/pt-br/acompanhe-oplanalto/noticias/2020/06/governo-federal-prorroga-o-auxilio-emergencial.

5 Nota del Ministro de Economía de Brasil del 30 de junio de 2020. 
vidades creativas, especialmente las relacionadas con las festividades artísticas y culturales, conducen a la inclusión de minorías generalmente excluidas como las mujeres y jóvenes.

\section{Solidaridady ciudadanía}

Paralelamente a la innovación digital, ha surgido un sentimiento de solidaridad que ha movilizado a la sociedad civil. Los ciudadanos han comenzado a actuar de manera colectiva en respuesta a las necesidades de las comunidades vulnerables. Las empresas del sector privado de todos los tamaños se han comprometido más con la responsabilidad social. Las empresas están más atentas no solo para satisfacer la demanda de los clientes, sino también para ser más sensibles al impacto socioeconómico de sus actividades a nivel local. Las empresas, organizaciones sin ánimo de lucro y particulares han distribuido paquetes de ayuda que incluyen cestas de alimentos básicos, productos de higiene y mascarillas.

Diariamente, las noticias de televisión presentaban una lista de proyectos, campañas e iniciativas creativas para ayudar a quienes lo necesitan. Un ejemplo es el proyecto Mesa Brasil del SESC-RJ (SESC) ${ }^{6}$, que se ha dedicado a combatir el hambre y reducir el desperdicio de alimentos. El proyecto recolecta donaciones de alimentos para los más pobres mientras los educa sobre cómo preparar alimentos más saludables. También hay un vínculo entre estos esfuerzos y las instituciones culturales, pues el público de las representaciones teatrales y los espectáculos presentados en los teatros del SESC (antes y después del aislamiento social) pueden obtener precios de entradas más baratos si llevan comida para donar. Este proyecto, que ya existía, se amplió durante el periodo COVID-19. Otro proyecto de SESC es

6 Mesa Brasil, SESC: https://www.sesc.com.br/portal/site/mesabrasilsesc/home.

Dos Santos-Duisenberg, Edna (2021). «Economía creativa, cultura y educación en tiempos de pandemia». Monograma. Revista Iberoamericana de Cultura y Pensamiento, n. ${ }^{\circ}$ 9, pp. 335-349. doi: 10.36008/monograma.2021.09.2235. http: revistamonograma.com. ISSN: 2603-5839. 
\#MesaSemFome a través del cual personalidades conocidas y artistas donan su tiempo, conocimiento y experiencia para apoyar la solidaridad de muchas maneras diferentes: llamando a personas mayores para contarles historias, haciendo sus compras, dando lecciones de instrumentos musicales y mejorando las habilidades culinarias, etc. Cada semana se ofrecen muchas actividades a través de Lives Solidarias de Instagram.

La inteligencia artificial y la robótica también tuvieron un papel en la lucha contra la pandemia. Con una población de 217 millones de personas, Brasil no tiene un número adecuado de test de COVID-19 para todos sus habitantes. Para hacer frente a esta situación de déficit, el Ministerio de Salud ha utilizado robots para llamar a las personas mayores con alto riesgo de contagio para un breve diagnóstico por teléfono. La plataforma TeleSUS ${ }^{7}$ desde abril 2020 ha iniciado el monitoreo del flujo de contagio con el objetivo de llegar a millones de personas a través de una búsqueda activa por teléfono y consultas por telemedicina. Aunque esta iniciativa no ha sido suficiente dada la expansión de la pandemia, la iniciativa ha sido positiva para mejorar el sentimiento de ciudadanía y autoestima de la población.

\section{Respuestas de política culturaly de economía creativa en Brasil}

En términos de cultura, todos los espacios culturales como cines, teatros y museos se han cerrado y los eventos que incluyen espectáculos artísticos, festivales y exposiciones se suspendieron a mediados de marzo de $2020^{8}$, para cumplir con las medidas de distanciamiento social durante la pandemia. El arte y la cultura aportan $\mathrm{R} \$ 170$ mil millones 9 anuales a la economía brasileña, proporcionando empleos directos e indirectos a cerca de cinco

\footnotetext{
7 TELESUS: https://aps.saude.gov.br/ape/corona/telesus.

8 Comunicado conjunto del Ministerio de la Salud y la Secretaría de Cultura y Economía Creativa.

9 Datos del Instituto Brasileiro de Geografia e Estatística.
} 
millones de personas que representan casi el $6 \%$ de la fuerza laboral nacional. Artistas, productores culturales, técnicos y profesionales creativos fueron los primeros en detener sus actividades como consecuencia de la pandemia y probablemente serán los últimos en reiniciarse, lo que los convierte en una de las categorías más afectadas. Por lo tanto, el Congreso aprobó una Ley de Emergencia Cultural ${ }^{10}$ (Lei Aldir Blanc) que ha permitido el uso de recursos del Fondo Cultural Federal ( $\mathrm{R} \$ 3$ mil millones) para proporcionar auxilio de emergencia durante tres meses para ayudar a compensar la pérdida de ingresos y proporcionar exención de impuestos de hasta seis meses para las industrias culturales y las empresas creativas.

Las pautas para la implementación de proyectos culturales durante la pandemia COVID-19 han sido revisadas. Los proyectos deben estar bien documentados y los productores deben proporcionar evidencia de cada acción tomada, en particular para proyectos financiados por la Ley de Incentivo a la Cultura (Lei Roanet). Se diseñaron tres medidas para aliviar el impacto de la pandemia y guiar la ejecución de los proyectos: 1) se permitirá a los proyectos utilizar hasta el 20\% del capital estimado; 2) los proyectos ahora pueden modificarse en cualquier momento (anteriormente había un límite); 3) la evaluación del proyecto será más flexible en la forma y el uso de los recursos.

Además, se adoptaron medidas especiales relacionadas con la cancelación de servicios y eventos en las áreas de turismo y cultura durante la pandemia. Las medidas abarcan cines, teatros, plataformas digitales, artistas y todos los profesionales contratados para trabajar en eventos y espectáculos culturales. Los afectados por el bloqueo que no los pudieron realizar, tendrán hasta un año para proporcionar los servicios ya contratados.

10 Lei Aldir Blanc:

https://www.in.gov.br/en/web/dou/-/lei-n-14.017-de-29-de-junho-de-2020-264166628.

Dos Santos-Duisenberg, Edna (2021). «Economía creativa, cultura y educación en tiempos de pandemia». Monograma. Revista Iberoamericana de Cultura y Pensamiento, n. ${ }^{\circ}$ 9, pp. 335-349. doi: 10.36008/monograma.2021.09.2235. http: revistamonograma.com. ISSN: 2603-5839. 
Para el Estado de São Paulo, las industrias culturales y creativas representan el 4\% del PIB. En 2020, la pérdida en el estado causada por COVID-19 fue estimada en $\mathrm{R} \$ 34,5 \mathrm{mil} \mathrm{mi-}$ llones y más de 650 mil personas se han quedado sin ingresos. Se ofreció una línea de crédito de $\mathrm{R} \$ 500$ millones para pymes y $\mathrm{R} \$ 150$ millones para microcréditos con condiciones especiales para micro, pequeñas y medianas empresas en los sectores cultural y creativo ${ }^{11}$. Además, el Festival \#CulturaemCasa fue una plataforma lanzada por la Secretaría de Cultura y Economía Creativa de São Paulo para estimular el distanciamiento social y al mismo tiempo mejorar el acceso a los contenidos culturales virtuales ofrecidos por instituciones culturales públicas. A través de la plataforma, el público puede visitar espectáculos, conciertos, museos, charlas, conferencias, leer libros y ver películas y obras de teatro. Hay muchas opciones diferentes para una variedad de edades e intereses, y el contenido está disponible y es actualizado diariamente. Esta plataforma de transmisión tuvo éxito al alcanzar 850 mil visitas en dos meses y acoger internautas de 107 países $^{12}$. Todo el contenido cultural permanecerá disponible durante el bloqueo de COVID-19.

La Secretaría de Cultura y Economía Creativa de Brasilia ha formalizado un esquema de financiación de $\mathrm{R} \$ 750$ millones $^{13}$ para ayudar a artistas locales y profesionales creativos culturales afectados por la cancelación de festivales y espectáculos culturales. El esquema proporciona tres líneas de crédito diferenciadas para microempresas, artistas independientes, así como préstamos e inversiones para apoyar a las pymes culturales y creativas. La Secretaría de Cultura y Economía Creativa del Estado de Río

11 Secretaria de Cultura e Economia Criativa, São Paulo.

12 Secretaria de Cultura e Economia Criativa, São Paulo: https://www.cultura.sp.gov.br/ culturaemcasa-completa-dois-meses-com-mais-de-850-mil-visualizacoes.

13 Secretaria de Cultura e Economia Criativa, Brasília:

http://cultura.ri.gov.br/governo-do-estado-lanca-edital-para-producoes-online. 
de Janeiro lanzó una oferta oficial para proyectos de producción cultural en línea. \#culturapresente recibirá $\mathrm{R} \$ 3,7$ millones del Fondo Estatal para la Cultura ${ }^{14}$. Cubrirá música, literatura, artes visuales, audiovisuales, danza, teatro, circo, moda, museos, comida cultural típica y nuevas expresiones culturales populares. Otro proyecto, «Cuentacuentos por teléfono», llamó a los voluntarios a contactar a personas mayores y personas que viven solas para contar historias, como una forma de minimizar el sentimiento de soledad. Esto permite que poetas, músicos y narradores se comprometan ofreciendo esperanza y solidaridad a las personas solitarias.

\section{Experiencias culturales en la era digital en Brasil}

Han surgido también iniciativas creativas de artistas e instituciones, y es probable que algunas permanezcan en la fase pospandemia. Dos tendencias fuertes han marcado la pandemia: la solidaridad y la transmisión de medios en vivo streaming: la combinación de transmisión en vivo y solidaridad ha dado como resultado las «Vidas Solidarias». En Brasil, más de 120 programas en línea recaudaron $\mathrm{R} \$ 17,6$ millones $^{15}$ en donaciones para combatir la COVID-19 en comunidades pobres. La movilización de artistas ha aportado innovación y es una forma de implicar a celebridades junto con individuos de la sociedad civil en causas sociales.

Los conciertos en vivo como \#tamojunto se convirtieron en la fiebre del sábado noche durante la pandemia. Los mejores cantantes brasileños (particularmente cantantes de música

14 Secretaria de Cultura e Economia Criativa, Rio de Janeiro:

http://cultura.ri.gov.br/governo-do-estado-lanca-edital-para-producoes-online.

15 Jornal Folha de São Paulo, junio 2020:

https://www1.folha.uol.com.br/empreendedorsocial/2020/06/lives-levantaram-r-176-

milhoes-em-doacoes-durante-a-pandemia.shtml. 
country) se presentan en casa, atrayendo una gran audiencia virtual y millones de «me gusta» en YouTube e Instagram. Entre los 10 conciertos en vivo más concurridos en todo el mundo, siete son de artistas brasileños. Marilia Mendonça, que recibió 3,31 millones de «me gusta», ocupó el puesto número uno a nivel mundial, seguida de Jorge y Mateus, con 3,24 millones ${ }^{16}$. Esto se explica en parte por el hecho de que el $70 \%$ de la música que se consume en Brasil se produce localmente. Además, el país ocupa el tercer lugar entre los principales productores de contenido digital creativo y como consumidores de servicios digitales.

Durante la pandemia, los festivales en línea como el Festival EuFicoEmCasa ofrecen entretenimiento a las personas a través de las redes sociales. A medida que se cancelaron los espectáculos y conciertos, los músicos y artistas visuales están trabajando virtualmente para brindar entretenimiento y expandir su audiencia y red a través de Instagram y YouTube. El primer festival reunió a 78 artistas, ofreciendo más de 40 horas de música durante el primer fin de semana en casa. Gracias a su éxito, se utiliza el mismo formato para festivales que ahora tienen lugar todos los fines de semana.

Evidentemente, después de más de un año de distanciamiento social, el sector cultural y las industrias creativas, sin actividad cotidiana, tuvieron que reinventarse en su lucha por la supervivencia. Paradójicamente, el consumo cultural en línea y la producción creativa están aumentando. La música lidera modelos innovadores con conciertos en vivo, pero las compañías de teatro también producen obras de teatro para presentaciones web sin público. Los cines drive-in están de vuelta. Los festivales virtuales de cortometrajes están atrayendo un nuevo público. Los libros electrónicos y una nueva generación de videojuegos inte-

16 Jornal O Globo, mayo 2020: https://oglobo.globo.com/cultura/lives-de-2020-saodominadas-por-brasileiros-com-sete-das-10-maiores-audiencias-no-mundo-24430222. 
ligentes tienen una gran demanda. Las subastas de arte visual y callejero están atrayendo a los amantes de la cultura, y el público televisivo ha aumentado con la repetición de antiguas telenovelas y producciones de pequeño formato.

Canales web, podcasts, streaming, donaciones conscientes, producciones creativas de colaboración híbrida, crowdfunding virtual público y privado son algunas alternativas emergentes. Ante tantas novedades ciertamente hay más preguntas que respuestas. Como las transmisiones en vivo están utilizando plataformas sociales que fueron diseñadas para ser efímeras, ¿'sobrevivirán las experiencias culturales en vivo? ¿Cómo nos aseguramos de que las producciones culturales en línea resistan la búsqueda continua de novedades? Si se cierra una plataforma social, ¿desaparecerá todo su contenido cultural? Los artistas famosos están encontrando grandes patrocinadores, pero la gran mayoría de los artistas están ofreciendo sus servicios por tarifas pequeñas o gratuitas. ¿Cómo nos aseguramos de que los artistas y las instituciones culturales puedan sobrevivir a largo plazo? Más que nunca, se necesita creatividad para optimizar la digitalización y encontrar formas de monetización factible y sostenible. Las circunstancias actuales son un desafío y el futuro es incierto, pero el arte y la cultura siempre encontrarán su camino en la sociedad contemporánea.

\section{La cultura, el ocio y el bienestar}

Paradójicamente, el cambio del comportamiento humano durante la pandemia nos devolvió a una teoría de la economía que surgió hace un siglo (Ruskin, 1900): «No hay riqueza sino vida». En este contexto nuevo-viejo, el tiempo libre, el ocio, el bienestar y la cultura están estrechamente asociados ${ }^{17}$. Normalmente, usamos nuestro tiempo libre para realizar actividades que no están

17 E. Dos Santos-Duisenberg, octubre 2020, «Of here and now: Pandemic \& Society in 2020». Modern Diplomacy, EU, octubre 2020. 
directamente relacionadas con el trabajo, deberes u ocupaciones domésticas. El ocio, sin embargo, es un concepto subjetivo que varía en función de la sociedad a la que pertenecemos. Está relacionado con nuestra participación en la vida cultural, reflejando los valores y características de una nación. Por tanto, puede considerarse un derecho humano según la Declaración de Derechos Humanos de la ONU (1948), y en particular la Convención Internacional de 1967 sobre Derechos Económicos, Sociales y Culturales ${ }^{18}$.

Durante la pandemia, hemos reflexionado más sobre la importancia del bienestar y la espiritualidad. Es innegable que las limitaciones provocadas por las medidas de encierro y el distanciamiento social nos ofrecieron más tiempo libre, pero opciones de ocio muy limitadas. Sin embargo, ganamos tiempo adicional para estar más cerca de nuestros seres queridos. Disfrutar de la vida familiar, incluso comer y cocinar juntos, se convirtió en un placer compartido y un nuevo estilo de vida.

Los momentos de ocio son imprescindibles en todas las fases de nuestra vida. Durante la infancia y la adolescencia, la mayor parte de nuestro tiempo se dedica al estudio y al deporte, mientras que durante la edad adulta nuestro tiempo se dedica principalmente al trabajo y la familia. De hecho, es en la vejez cuando las personas tienen más tiempo libre para disfrutar de eventos culturales, ocio y turismo. A nivel mundial, las personas son más longevas y, por consiguiente, se está configurando una nueva estructura de edades: los jóvenes mayores (65-74 años), los intermedios (75-84 años) y los mayores (a partir de los 85 años). Según las Naciones Unidas, en 2018, por primera vez en la historia, las personas de 65 años o más superaron en número a los niños menores de cinco años.

18 ONU, Convención sobre Derechos Económicos, Sociales y Culturales: https://treaties.un.org/doc/treaties/1976/01/19760103\%2009-57\%20pm/ch_iv_03.pdf. 
Además, en un corto periodo de tiempo, muchos pasaron de un estilo de vida social y cultural muy activa hacia una vida sencilla. Debido a las limitaciones de distanciamiento social y vida al aire libre, las actividades de tiempo libre tuvieron que ser menos deportivas (sin bicicleta, tenis, jogging, etc.) y más creativas, como diseñar, pintar y escribir, cocinar. Las pandemias globales también afectan a nuestra salud mental colectiva. Ante la inseguridad sanitaria y económica imperante, el foco ha estado en el bienestar, fortalecer las amistades, ampliar nuestras redes sociales, practicar la solidaridad, mejorar la autoestima, así como reflexionar sobre la espiritualidad y la religión.

Durante los momentos de tensión de la pandemia, la gente ha quedado emocionalmente frágil. Las personas están sufriendo pérdidas que persistirán mucho después de que termine la pandemia. Algunos se sienten estresados o deprimidos, mientras que otros reaccionan buscando alivio en el ejercicio, la relajación, la meditación, el yoga o el entrenamiento de la atención plena mindfulness. En Brasil, en el Día Internacional de la Paz, el 21 de septiembre de 2020, el festival Virada Zen ${ }^{19}$ celebró la cultura de la paz con una meditación virtual global y simultánea, con la participación de más de 200 líderes espirituales y maestros que ofrecieron una experiencia de conexión y bienestar a través de prácticas como meditación, cultura de paz y yoga, hablando también de la importancia de la diversidad y el desarrollo humano.

Por otro lado, una investigación reciente llevada a cabo en el Reino Unido ${ }^{20}$ para rastrear el consumo cultural digital durante la pandemia ha indicado que la media del tiempo que se pasa diariamente viendo televisión fue de cuatro horas, mientras

19 Festival Virada Zen, São Paulo, 2020: https://www.viradazen.com.br/conteudo/o-que-e-avirada-zen.html.

20 AudienceNet para la Oficina de Propiedad Intelectual y PEC (Centro de Políticas y Evidencias de las Industrias Creativas) en el Reino Unido: https://www.pec.ac.uk/policy-briefings/ digital-culture-consumer-panel. 
escuchar música, ver películas y jugar videojuegos cada día fueron tres horas respectivamente. Comprender el comportamiento humano, en particular los hábitos de los más jóvenes, es un parámetro esencial para indicar nuevas tendencias culturales y consolidar la cohesión social en tiempos posteriores a una pandemia. Igualmente, estas evidencias serán útiles para la formulación de políticas de reanudación pospandemia, incluso para incitar los gobiernos a involucrar a las instituciones culturales y emplear artistas y creativos para estimular el proceso de curación colectiva y reactivar la recuperación cultural y socioeconómica.

Es ampliamente reconocido que los sectores de las artes, la cultura y la creatividad se vieron muy afectados por la pandemia. Mientras que los productos culturales y creativos digitales para el consumo doméstico tenían una gran demanda, otros bienes creativos tangibles como los productos de arte, artesanía, moda y diseño sufrieron una fuerte contracción. Muchos artistas y creativos no tuvieron otra opción que experimentar el trabajo en espacios digitales.

A pesar de que 4,5 billones de personas $(60 \%$ de la población mundial) utilizan internet, la disponibilidad de un acceso de banda ancha a precios asequibles es una condición previa para que todos puedan beneficiarse de las oportunidades que brindan las herramientas digitales. Esto se aplica tanto a los productores como a los consumidores de contenido digital cultural y creativo. En la actualidad, los vídeos representan el 80-90\% de la circulación global de datos digitales, pero al mismo tiempo, América Latina, Medio Oriente y África juntos representan solo el 10\% del tráfico de datos mundial ${ }^{21}$. Esta evidencia apunta a que las asimetrías digitales se están agravando. La creatividad por sí sola no es suficiente para transformar ideas en productos o servicios creativos comercializables si no se dispone de infraestructura y herramientas digitales.

21 UNCTAD, Digital Economy Report 2019.

Dos Santos-Duisenberg, Edna (2021). «Economía creativa, cultura y educación en tiempos de pandemia». Monograma. Revista Iberoamericana de Cultura y Pensamiento, n. ${ }^{\circ}$ 9, pp. 335-349. doi: 10.36008/monograma.2021.09.2235. http: revistamonograma.com. ISSN: 2603-5839. 


\section{Educación, aprendizaje continúa y conectividad}

La pandemia obviamente tuvo un impacto significativo en la educación y el aprendizaje. Repensar la educación ya era un tema en la agenda de muchos países para responder a los desafíos actuales del mercado laboral. Además de la necesidad de adaptar la metodología y las prácticas pedagógicas, muchos creen que es necesario traer un enfoque interdisciplinario y aplicado a los currículos con enfoque en ciencia, tecnología, ingeniería y matemáticas (STEM), preferiblemente integrando también las artes (STEAM). De todas maneras, el sistema educativo se ha visto obligado a adaptarse rápidamente al aprendizaje a distancia. A nivel mundial, más de 1,2 billones de niños estuvieron fuera del aula en 186 países durante casi todo el periodo de la pandemia ${ }^{22}$. En América Latina, las escuelas estuvieron cerradas y más de 154 millones de niños entre 5 y 18 años se quedaron en casa en lugar de ir a clase durante casi un año y medio ${ }^{23}$. Además, el acceso al material escolar se distribuye de manera desigual; los estudiantes más ricos tienen acceso a internet y la educación en el hogar, mientras que los más pobres no. En algunos países programas televisivos están facilitando la educación a distancia. Pero los jóvenes están perdiendo meses de aprendizaje y esto tendrá efectos duraderos. La pérdida de capital humano en el futuro será enorme.

En el lado positivo, el aprendizaje continuo se ha convertido en una tendencia y una necesidad. La innovación y la adaptación digital dieron lugar a una amplia gama de cursos online. Millones de estudiantes están mejorando sus conocimientos y habilidades en diferentes dominios a través del aprendizaje a distancia, ya sea a través de aplicaciones de idiomas y música, videoconferencias o aprendizaje de software. Algunos cursos son gratuitos, otros tie-

22 World Economic Forum.

23 Banco Interamericano de Desarrollo.

Dos Santos-Duisenberg, Edna (2021). «Economía creativa, cultura y educación en tiempos de pandemia». Monograma. Revista Iberoamericana de Cultura y Pensamiento, n. ${ }^{\circ}$ 9, pp. 335-349. doi: 10.36008/monograma.2021.09.2235. http: revistamonograma.com. ISSN: 2603-5839. 
nen que pagarse, con o sin certificación, y en diferentes niveles, pero lo absolutamente transformador es que el acceso al conocimiento se ha vuelto más democrático. Independientemente de la edad o el campo de interés, los estudiantes de diferentes partes del mundo pueden tener acceso a universidades de prestigio o formación práctica. El aprendizaje electrónico, en el que la enseñanza se realiza de forma remota y en plataformas digitales, ya existía, pero la demanda ha aumentado considerablemente durante la pandemia y este podrá ser un punto sin retorno.

Después de un año de pandemia hay signos contundentes de que la década de 2020 enfrentará un nuevo conjunto de desafíos y la vida no volverá a ser como antes. El futuro cercano será muy diferente en comparación con el pasado reciente. Es probable que la esperanza y el miedo coexistan durante cierto tiempo. Hay nuevos valores, nuevos estilos de vida, nuevos comportamientos sociales, nuevos estándares de consumo y nuevas formas de trabajar y estudiar. La pandemia ha impuesto una profunda reevaluación ética y filosófica en la sociedad. Este punto de inflexión está conduciendo a una profunda renovación socioeconómica y, con suerte, a una sociedad más inclusiva y sostenible. 